\title{
Running applications on a hybrid cluster
}

\author{
A. V. Bogdanov ${ }^{1, a}$, I. G. Gankevich ${ }^{1}$, V. Yu. Gayduchok ${ }^{2}$, N. V. Yuzhanin ${ }^{1}$ \\ ${ }^{1}$ Saint Petersburg State University, University ave. 35, St. Petersburg, Peterhof, 198504, Russia \\ ${ }^{2}$ Saint Petersburg Electrotechnical University "LETI", St. Professora Popova 5, St. Petersburg, 197376, Russia \\ E-mail: ${ }^{a}$ bogdanov@csa.ru
}

Received December 17, 2014

A hybrid cluster implies the use of computational devices with radically different architectures. Usually, these are conventional CPU architecture (e.g. x86_64) and GPU architecture (e. g. NVIDIA CUDA). Creating and exploiting such a cluster requires some experience: in order to harness all computational power of the described system and get substantial speedup for computational tasks many factors should be taken into account. These factors consist of hardware characteristics (e.g. network infrastructure, a type of data storage, GPU architecture) as well as software stack (e.g. MPI implementation, GPGPU libraries). So, in order to run scientific applications GPU capabilities, software features, task size and other factors should be considered.

This report discusses opportunities and problems of hybrid computations. Some statistics from tests programs and applications runs will be demonstrated. The main focus of interest is open source applications (e. g. OpenFOAM) that support GPGPU (with some parts rewritten to use GPGPU directly or by replacing libraries).

There are several approaches to organize heterogeneous computations for different GPU architectures out of which CUDA library and OpenCL framework are compared. CUDA library is becoming quite typical for hybrid systems with NVIDIA cards, but OpenCL offers portability opportunities which can be a determinant factor when choosing framework for development. We also put emphasis on multi-GPU systems that are often used to build hybrid clusters. Calculations were performed on a hybrid cluster of SPbU computing center.

Keywords: GPGPU, HPC, computational clusters, OpenFOAM, LINPACK, ViennaCL, CUDA, OpenCL

The research was carried out using computational resources of Resource Center Computational Center of Saint Petersburg State University (T-EDGE96 HPC-0011828-001) and partially supported by Russian Foundation for Basic Research (project No. 13-07-00747) and Saint Petersburg State University (project No. 9.38.674.2013)

Citation: Computer Research and Modeling, 2015, vol. 7, no. 3, pp. 475-483. 


\title{
Запуск приложений на гибридном кластере
}

\author{
А. В. Богданов ${ }^{1}$, И. Г. Ганкевич ${ }^{1}$, В. Ю. Гайдучок ${ }^{2}$, Н. В. Южанин ${ }^{1}$ \\ ${ }^{1}$ Санкт-Петербургский государственный университет, Россия, 198504, г. Санкт-Петербург, Петергоф, \\ Университетский просп., д. 35 \\ ${ }^{2}$ Санкт-Петербургский государственный электротехнический университет «ЛЭТИ», Россия, 197376 , \\ г. Санкт-Петербург, ул. Профессора Попова, д. 5
}

Гибридный кластер подразумевает использование вычислительных ресурсов с различными архитектурами. Как правило, в таких системах используется CPU распространенной архитектуры (например, x86_64) и GPU (например, NVIDIA CUDA). Создание и эксплуатация подобного кластера требует определенного опыта: для того чтобы задействовать все вычислительные мощности такой системы и получить существенное ускорение на задачах, требуется учесть множество факторов. К таким факторам относятся как характеристики оборудования (например, особенности сетевой инфраструктуры, хранилища, архитектуры GPU), так и характеристики программного обеспечения (например, реализация МPI, библиотеки для работы с GPU). Таким образом для эффективных научных расчетов на подобных системах требуется помнить о характеристиках ускорителя (GPU), особенностях программного обеспечения, характеристиках задачи и о многих других факторах.

В этой статье анализируются достоинства и недостатки гибридных вычислений. Будут приведены результаты запуска некоторых тестов и научных приложений, использующих GPGPU. Ocновное внимание уделено программных продуктах с открытым исходным кодом, которые поддерживают работу c GPGPU.

Существует несколько подходов для организации гетерогенных вычислений. В данной статье мы рассмотрим приложения, использующие CUDA и OpenCL. CUDA довольно часто используется в подобных гибридных системах, в то время как переносимость OpenCL-приложений может сыграть решающую роль при выборе средства для разработки. Мы также уделим внимание системам с несколькими GPU, которые все чаще используются в рамках подобных кластеров. Вычисления проводились на гибридном кластере ресурсного центра «Вычислительный центр СПбГУ».

Ключевые слова: GPGPU, высокопроизводительные вычисления, вычислительные кластеры, OpenFOAM, LINPACK, ViennaCL, CUDA, OpenCL 


\section{Introduction}

Recent years have shown growing interest to hybrid computations. It became clear that conventional architectures have limited performance, which is in many cases inferior to performance of hybrid ones, let alone energy consumption and heat generation [Huang, Xiao, Feng, 2009]. Today one can face many different hybrid architectures, the vast majority of them are usually employ SIMDaccelerator (GPU, Cell, MIC, etc.) and a conventional CPU. This report concerns GPGPU as one of the earliest accelerator implementation.

One should clearly understand that such systems are not a panacea: while there are many tasks that can be smoothly mapped on GPGPU there are some classes of algorithms that can not benefit from implementing them for GPGPU. This report concerns aspects of running applications on a hybrid cluster that contains several GPGPUs on each node.

One can look at TOP-500 list and find out that the most powerful supercomputers are hybrid clusters: today (June 2014 TOP-500 list) about $35 \%$ of overall performance of TOP-500 list systems is given by some accelerator extension cards [TOP 500 list statistics...] which is almost four times more than in 2010 (hybrid systems provided only $9 \%$ of total performance at that time).

It can be explained with a simple fact: hybrid systems are constantly evolving that leads to performance growth while preserving and improving GFLOPS/Watt ratio. Software for hybrid systems is improving too. Software companies develop new libraries for GPGPU and applications that use such libraries, there are already several standards for GPGPU (e. g. OpenCL, OpenACC).

Such evolution can be seen on the example of GPGPU and other accelerators. Manufacturers try to ease programming of such systems. Some compilers can automatically split tasks between CPU and GPU. In case of MIC there are two basic approaches: native compilation and offload when MKL automatically offloads parts of a program to accelerator [Intel Xeon Phi Coprocessors...].

But one should clearly understand that not all tasks benefit from GPGPU usage (some tasks can show speedup while others show even slowdown when running on GPGPU) and remember that this area is still developing, so it will probably take time for such systems to become quite common and quite simple for programming.

\section{GPU use cases}

There are several ways one can harness GPU.

Conventional usage. The obvious case. GPU is used for graphics computations, relatively simple. One or multiple GPUs per one node. Probably there is no need for building a cluster in that case, since compute-intensive calculations are a part of the third case.

GPUs for virtual machines. The next approach is to use some GPUs within one or several virtual machines. Virtualization technologies are wide-spread due to advantages they offer to organizations and end users. Virtual CPU and network devices become ubiquitous while virtualization of powerful GPUs is an actively developed area which becomes a point of interest for major manufacturers. Such techniques are implemented, for example, in VMWare products and XenServer. There are two basic approaches in this area.

- Dedicated GPU (GPU pass-through). This variant is similar to conventional GPU usage: hypervisor just gives a virtual machine unrestricted access to the whole GPU. So, such virtual machine can use the whole GPU, while other VMs have no access to this GPU.

- Virtual GPU. Fully virtualized GPU is used in this case. Such approach can be exemplified by NVIDIA GRID K2 GPU managing by XenServer. Such GPU can be divided into several virtual GPUs with different characteristics. Each virtual GPU can be assigned to a separate virtual machine.

A scheme in Figure 1 depicts this two approaches.

GPUs within computational clusters. Finally, one can create cluster which nodes will contain one or more GPGPUs. Such clusters are usually referred as hybrid clusters. While the second case 
(especially virtual GPUs) is still not common this case is the most frequently used approach for scientific computations. The main issues that arise in this case are listed below.
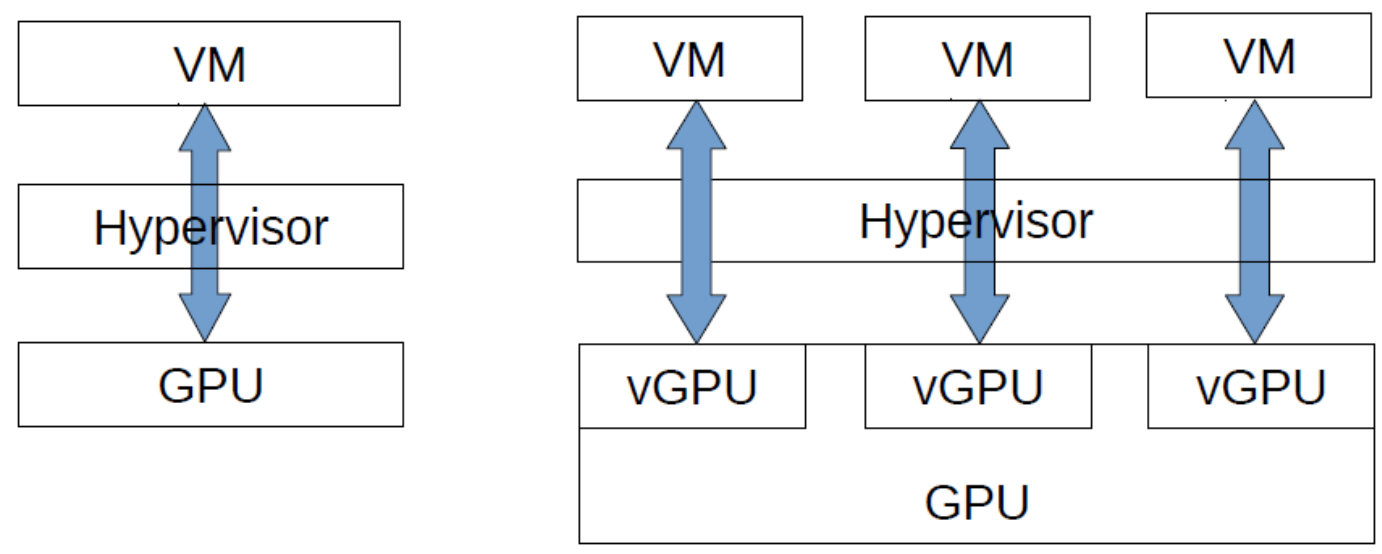

Fig. 1. Dedicated GPU and virtual GPUs

- How many GPUs should be installed into one node? The answer to this question is determined by several factors: node interconnect (that can be a "bottleneck" when the number GPUs is growing), the number of CPU cores (CPU will assign tasks to GPUs), etc.

- How to share cluster resources between users? One can solve this problem (it can be a real challenge in situation with limited resources that should be accessible for many users with radically different tasks) via Portable Batch System (PBS) [Torque resource manager]. There are several implementations of PBS that varies in some parameters, but the main idea is still the same: administrator creates several queues with different limitations. So, he can decide which users should have access to a particular queue.

- How to restrict user from using GPUs (or some other cluster resources) that assigned to another one? This question is solved by choosing appropriate managing system (for example, some reliable PBS implementations that can manage GPU access and so forth) and proper configuration as well.

\section{Platform specifications}

All tests for this report were performed using hardware of resource center of Saint Petersburg State University. This center has hybrid cluster with the following characteristics:

- 24 nodes;

- 2 CPU Intel Xeon X5650 (6 cores per CPU, total 12 cores);

- 96 RAM;

- 3 (16 nodes) or 8 ( 8 nodes) GPUs NVIDIA M2050 per node;

- Ethernet $10 \mathrm{G}$ network;

- Infiniband 4x QDR (40 Gbit/sec) network.

Peak performance of this complex is 59.6 TFLOPS. GPU peak performance is 0.5 TFLOPS (double precision), while CPU performance is 0.075 TFLOPS. Each node is running CentOS 6.4. Cluster management system is PBS.

\section{Basic tests}

For assessing the GPU performance and scalability of different tasks on GPU it's quite convenient to start from LINPACK. It is de facto standard benchmark for HPC systems (TOP-500 uses the best run of LINPACK test for creating the list). This test solves a dense system of linear 
equations using LU factorization. Figure 2 depicts the LINPACK test results (performance in GFLOPS for one node with 3 GPUs depending on the matrix size).

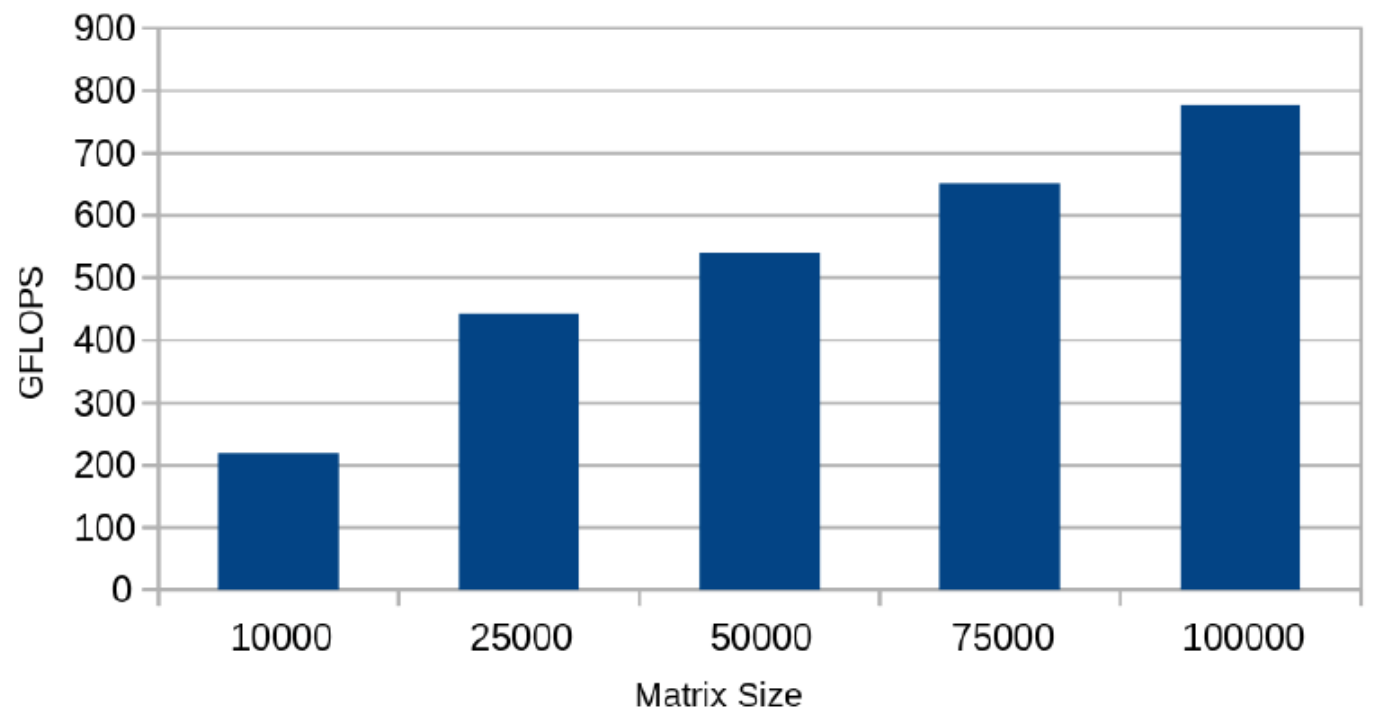

Fig. 2. LINPACK test results for nodes with 3 GPUs.

While LINPACK test uses double precision (TOP-500 takes into account only double precision LINPACK) such precision is not necessary for many tasks. We performed several tests that can be smoothly mapped on GPU (single and double precision). These tests show similar behavior: performance gain increases with task size. Coarse grained algorithms implementations show good scalability when run on multi-GPU systems. GPUs shows about twice as much performance for single precision in comparison to double precision for some tests. It is suits quite good to performance declared by manufacturer (0.5 TFLOPS for double and 1 TFLOPS for single precision). But these tests are synthetic, they can be efficiently mapped to GPGPU. Users don't need such tests (except for initial hardware testing) because they want to run real applications that solves their scientific problems. Some real world applications that uses GPGPU are discussed in the next section.

\section{Applications}

There are many applications that support GPGPU, open source and free, as well as commercial: Abinit, ANSYS, GROMACS, Matlab, OpenFOAM (with Ofgpu library or some other library for GPGPU), QuantumEspresso, etc. The list of such applications is constantly growing. We are interested in open source applications, but we will start from two well-known commercial applications because different scientists work with different applications (tools, libraries) and get used to different instruments, commercial and non-commercial, while our aim is to provide users with information about general rules for running GPGPU applications, point to a possible "bottlenecks", assess performance which can be gained on hybrid systems, we look at clusters from administrator perspective.

Figure 3 depicts the MATLAB 2011b BLAS level 3 test for single and double precision that was run on CPU and GPU.

This diagrams once again shows how task size influences the performance. Tasks that can be vectorized (matrix operations in this example) can be mapped to GPGPU and benefit from GPGPU usage.

The next example is ANSYS Fluent case. We decide to run small case on different number CPU cores and GPUs. ANSYS Fluent version 15 allows user to use both CPU and GPU within a run. The only rule is CPU cores number should be divisible by GPUs count (that's why for some configurations there is no time value). The results are depicted in Figure 4. 

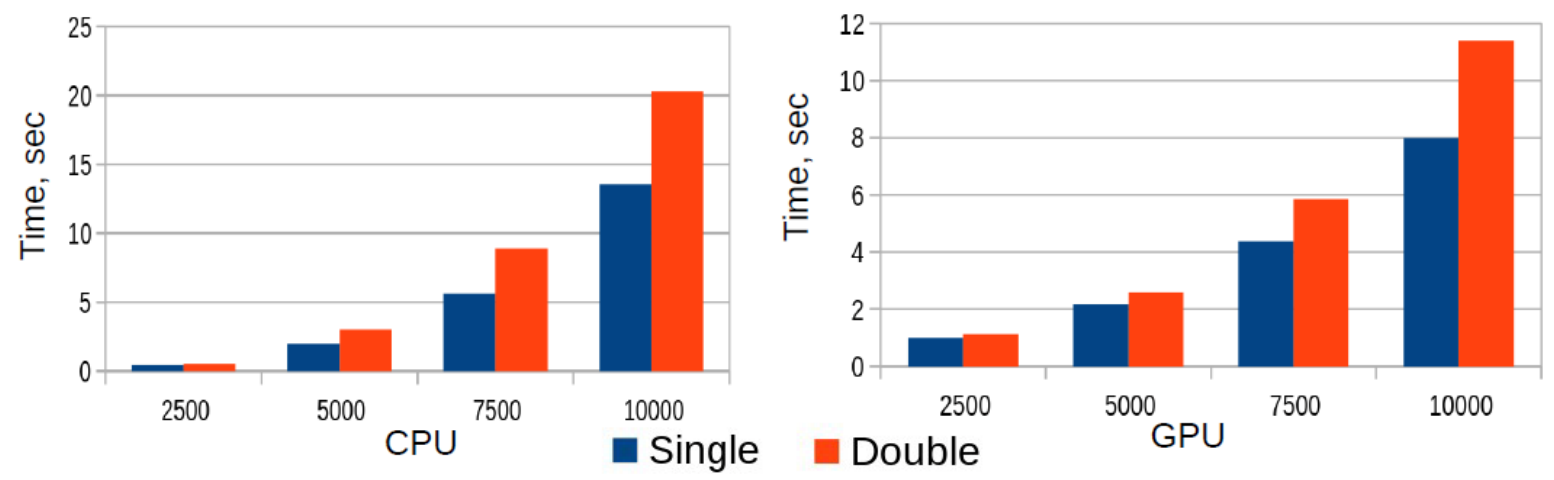

Fig. 3. MATLAB R2011b BLAS 3 level test

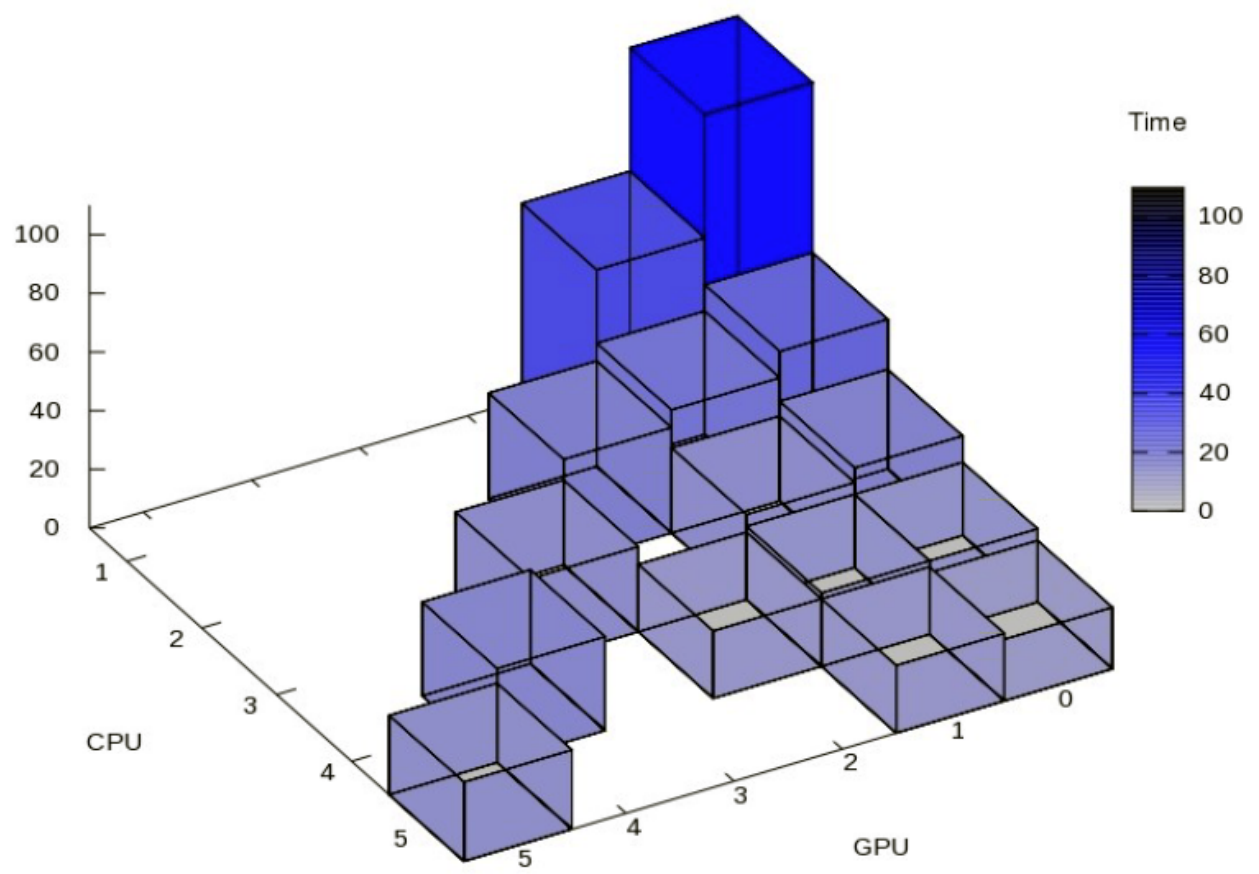

Fig. 4. ANSYS Fluent test results (small case)

As one can see small case has not so good scalability even if user runs certified large commercial product.

As an open source alternative to MATLAB we used ViennaCL in our tests. It has CUDA library and OpenCL versions and also version for MIC [ViennaCL...]. The results of LU decomposition test is depicted in Figure 5 (performance means here performance ratio, as compared with run time of the test with 500 matrix size).

And finally OpenFOAM. OpenFOAM is an open source platform for solving CFD problems [Jasak, Jemcov, Tukovic, 2007.]. There are several libraries for running OpenFOAM on GPU. Ofgpu is an open source library for GPU computations. It provides users with GPU linear system solvers and uses CUSP [GPU v1.1 Linear Solver Library...] to work with matrices.

There are several libraries for running OpenFOAM on GPGPU. Such libraries can be commercial or free. We decided to go for open source of gpu library.

OpenFOAM CFD calculations involve solving systems of linear equations. This task is usually offloaded onto GPUs.

Ofgpu provides users with 2 linear system solvers: PCGgpu (preconditioned conjugate gradient solver for symmetric matrices for GPGPU) and (PBiCGgpu - preconditioned biconjugate gradient 
solver for asymmetric matrices for GPGPU). These solvers should be specified in the file: $<$ case $>$ /sysem/fvSolution.

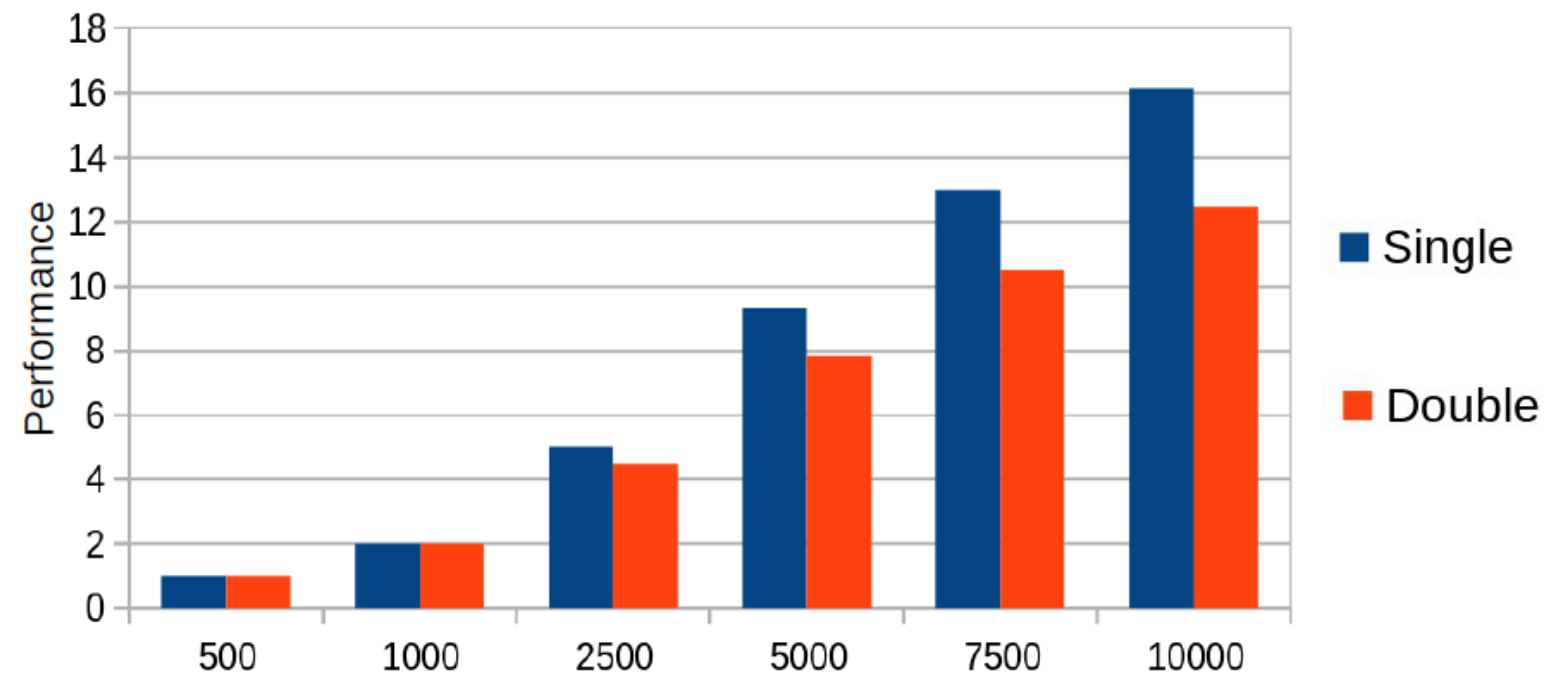

Fig. 5. ViennaCL LU decomposition test results

Ofgpu is built as a separate library and it should be loaded when OF solver use "* gpu" linear system solvers. User can specify GPU device to use in the file: <case>/sysem/controlDict ("cudaDevice" parameter).

Figure 6 and 7 depicts test results for CPU and GPGPU runs for different mesh sizes. Test case corresponds to a steady turbulent flow in a tube (OpenFOAM 2.2.2 single precision with OFGPU 1.1, compiled using Intel compilers (Intel Cluster Studio 2013; ICC 13.0.1), for CPU runs IMPI 4.1.0.024 from Intel Cluster Studio 2013 was used, CUDA Toolkit 5.5 was used for GPGPU version).

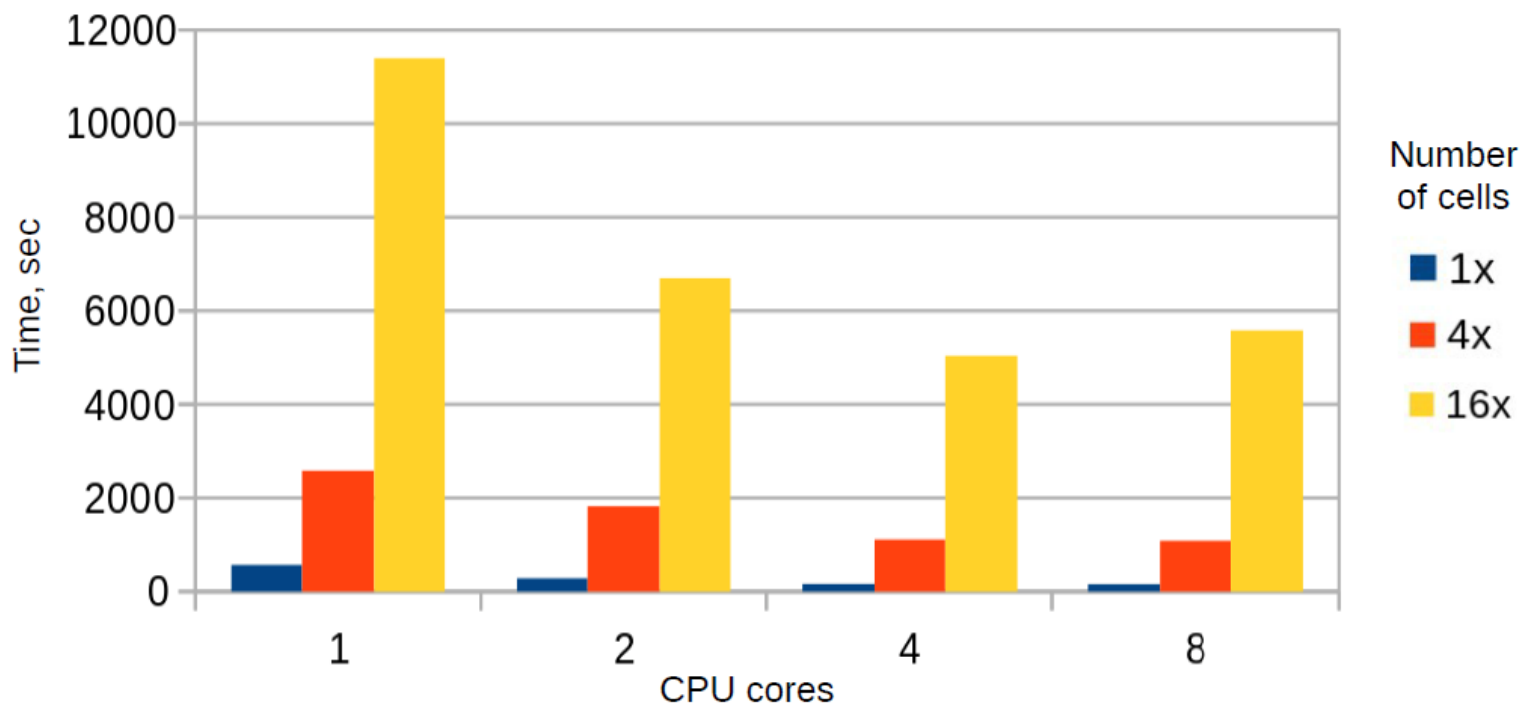

Fig. 6. OpenFOAM CPU test results

As one can see the finer mesh is used the more speedup is achieved.

But Ofgpu allows users to run OpenFOAM computations only on one GPU. Multi-GPU systems are not supported. One can run different tasks using different GPUs (by specifying GPU number), but one can not use several GPU within one task. We are currently working on multi-GPU version of ofgpu that will be able to harness multiple GPUs on different nodes on a hybrid cluster. The results will be published in future works. 


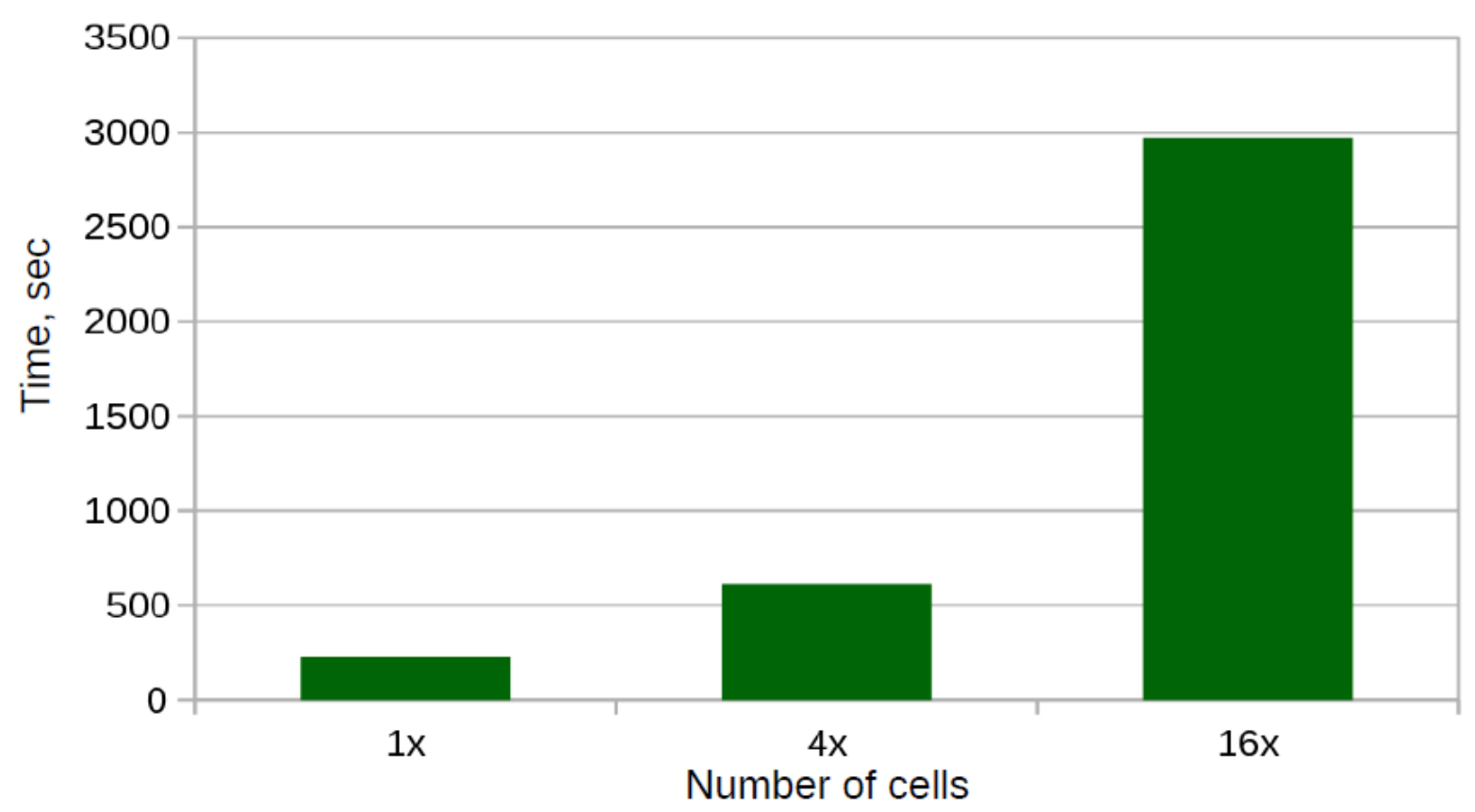

Fig. 7. OpenFOAM GPGPU test results

\section{Conclusions}

Hybrid clusters offer great peak performance, however, real performance depends on task. It is advisable to run coarse grained algorithms on such systems in order to decrease amount of data transferred between CPU and GPU because data transfer speed can be a bottleneck. In addition to this cluster architecture can introduce network speed as a new possible bottleneck. Software implementations can increase or decrease performance (for example, one should think about MPI implementation in case of MPI program). Performance gained is task size dependent. For example, one can get substantial speedup on GPU for CFD task when using finer mesh (but anyway there is a question whether or not one needs such fine mesh). Another possible problem is limited memory on GPU. When writing an application memory size should be taken into account. Programmer should choose the way he will use accelerators. He could write code for the accelerator using special API or delegate this work to compiler or special library that will offload the accelerator. The first method is advisable since compilers (or special libraries) are still can not split code for CPU and accelerators in a manner that will lead to great speedup. But programming such systems requires more accuracy from a programmer. Multi-GPU systems introduce new questions. And finally, one can get slightly different results on CPU and GPU working with floating point numbers. It concerns rounding and accordance to a floating point standard (e.g. IEEE 754) and it should be taken into account too.

Cluster management system can ease maintenance of such complexes. Sharing resources between users (especially GPUs) can be a challenge, but good management system can ease this task.

So, hybrid clusters are an actively developing area, promising systems that will probably be widely discussed subject in the future. Despite the fact that such systems introduce several issues that are not specific to traditional architectures they offer advantages that have never been specific to traditional architectures too.

\section{References}

GPU v1.1 Linear Solver Library for OpenFOAM, http://www.symscape.com/gpu-1-1- openfoam. 
Huang S., Xiao S., Feng W. On the Energy Efficiency of Graphics Processing Units for Scientific Computing. 2009

Intel Xeon Phi Coprocessors web pages, http://www.intel.com/

Jasak H., Jemcov A., Tukovic Z. Openfoam: A c++ library for complex physics simulations. International Workshop on Coupled Methods in Numerical Dynamics, IUC, Dubrovnik, Croatia, 2007.

Resource Center Computational Center Website, http://cc.spbu.ru

TOP 500 list statistics (category "Accelerator/Co-processor Performance Share"), http://www.top500.org/statistics/list/

Torque resource manager web pages, http://www.adaptivecomputing.com/products/opensource/torque/

ViennaCL web pages, http://viennacl.sourceforge.net/ 